\title{
Numerical Investigation of the Liquid Film Flows with Evaporation at Thermocapillary Interface
}

\author{
Ekaterina Rezanova 1, 2, a \\ ${ }^{1}$ Altai State University, Department of Informatics, pr. Lenina, 61, 656049 Barnaul, Russia \\ ${ }^{2}$ Kutateladze Institute of Thermophysics SB RAS, pr. Lavrentyeva, 1, 630090 Novosibirsk, Russia
}

\begin{abstract}
Flows of the thin liquid layers on an inclined non-uniformly heated substrate are investigated numerically. The evaporation at the thermocapillary interface is taking into account. The Oberbeck-Boussinesq equations and the generalized kinematic, dynamic and energy conditions on a thermocapillary boundary are used for governing equations. The evolution equation, which determines the position of the interface, is obtained on the basis of the long-wave approximation of the equations for moderate Reynolds numbers. The numerical algorithm for solving of this evolution equation is presented. Comparison of the numerical results of flows of various liquids is presented.
\end{abstract}

\section{Introduction}

The effects such as gravity and thermocapillary forces, additional tangential stresses and some other factors may affect the flows in thin liquid layers. The influences of various effects on the flow character are investigated analytically, numerically and experimentally [1-6]. Evaporation at the interface should be taken into account in the modeling of such processes [1-3, 7-9]. A number of works are devoted to the numerical study of flow of evaporating thin liquid layers [2-4]. The boundary conditions should provide a fulfillment of the conservation laws on the interface [10-12].

The motion of a viscous incompressible liquid film along an inclined at an angle $\alpha$ impermeable substrate is investigated numerically. Accompanying flow of gas moves over a liquid layer. The twodimensional mathematical model of the process is based on the Oberbeck-Boussinesq equations. The boundary conditions on thermocapillary interface are written with account of evaporation. Long-wave theory is applied to construct the nonlinear evolution equations for the liquid film thickness. This equation should incorporate the effects of gravity, viscosity, capillarity and thermocapillarity.

\section{The problem statement}

Let the interface position be determined by the equation $z=h(x, t)$. The equation defines the solid substrate has the form $z=0$, the $x$-axis coincides with the direction of fluid flow (see Fig.1). The gravity vector has the form $\mathbf{g}=\left(g_{1}, g_{2}\right)=(g \sin \alpha,-g \cos \alpha), g=|\mathbf{g}|$.

\footnotetext{
${ }^{\text {a }}$ Corresponding author : katerezanova@mail.ru
} 


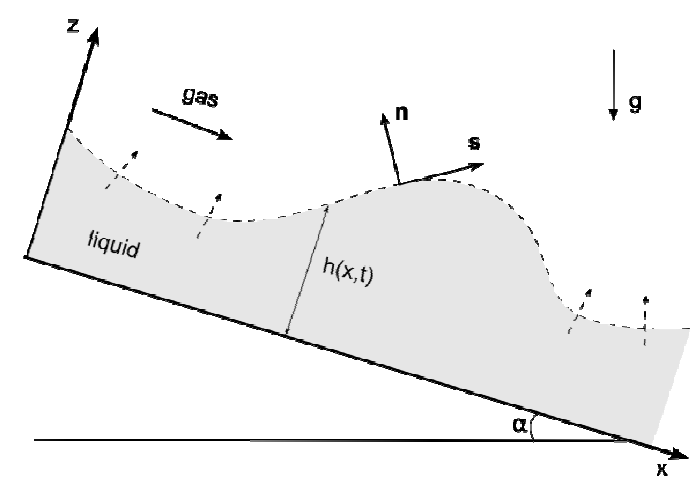

Figure 1. Geometry of flow domain

The one-sided mathematical model based on the Oberbeck-Boussinesq equations in the dimensionless form can be written as follows in the case, when the Reynolds number $R e=O(1)$ [7]:

$$
\begin{gathered}
\varepsilon^{2}\left(u_{t}+u u_{x}+w u_{z}\right)-\varepsilon^{2} u_{x x}=u_{z z}-p_{x}^{\prime}-\gamma_{1} T \sin \alpha, \\
\varepsilon^{4}\left(w_{t}+u w_{x}+w w_{z}\right)-\varepsilon^{4} w_{x x}-\varepsilon^{2} w_{z z}=-p_{z}^{\prime}+\gamma_{2} T \cos \alpha, \\
u_{x}+w_{z}=0, \\
\operatorname{Pr}^{2}\left(T_{t}+u T_{x}+T w_{z}\right)-\varepsilon^{2} T_{x x}=T_{z z} .
\end{gathered}
$$

Here $\varepsilon$ is the small parameter of the problem, $\varepsilon=d / l$ ( $d$ and $l$ are the transverse and longitudinal characteristic lengths), $\mathbf{v}=(u, w)$ is the velocity vector; $p^{\prime}$ is the modified pressure $\left(p^{\prime}=p-\gamma_{1}(\beta T *)^{-1} x\right.$ $\left.\sin \alpha+\gamma_{2}\left(\beta T_{*}\right)^{-1} z \cos \alpha\right) ; T$ is the temperature; $\rho$ - some relative value of the liquid density; $\operatorname{Pr}=v / \chi$ is the Prandtl number; $\gamma_{1}=G r / \varepsilon ; \gamma_{2}=G r ; G r=d^{3} g \beta T * v^{2}$ is the Grashof number; the Reynolds number is $R e=u * l / v ; v, \chi, \beta$ is the coefficients of the kinematic viscosity, thermal diffusivity and thermal expansion; $T *$ is the characteristic temperature drop; $u *$ is the characteristic longitudinal velocity of the liquid.

The generalized kinematic, dynamic and energy conditions $[7,12]$ can be written in the following dimensionless form after simplifications:

$$
\begin{gathered}
-\varepsilon\left(h_{t}+h_{x} u-w\right)=E J_{e v}, \\
u_{z}=\alpha_{\tau} \tau(x, t)-\alpha_{M a}\left(T_{x}+h_{x} T_{z}\right), \quad p=p^{g}-\alpha_{C a} h_{x x}\left(1-\alpha_{\sigma} T\right), \\
-T_{z}=\bar{\beta}_{3} J_{e v}+\bar{\beta}_{6} h_{x x} J_{e v} .
\end{gathered}
$$

Here $J_{e v}$ is the local mass flux at the thermocapillary interface, $E=\kappa T /\left(\lambda_{U} \rho v\right)$ is the evaporation parameter, $\kappa$ is the heat conductivity coefficient, $\lambda_{U}$ is the latent heat of vaporization, $\alpha_{\sigma}=\operatorname{MaCa} / \mathrm{Pr}$, $M a=\sigma_{T} T * l /(\rho v \chi)$ is the Marangoni number, $C a=u * \rho v / \sigma_{0}$ is the capillary number, $\sigma_{T}$ is the temperature coefficient of surface tension, $\sigma_{0}$ is the value of the coefficient of surface tension $\sigma$ at the some value of temperature (we assume the linear dependence of the surface tension on temperature $\sigma=\sigma_{0^{-}} \sigma_{T}(T$ $\left.T_{0}\right)$ ), $\alpha_{M a}=\operatorname{Ma\varepsilon } / \operatorname{Pr}, \alpha_{C a}=\varepsilon / C a, \alpha_{\tau}=\bar{\rho} \overline{v v} \varepsilon / \bar{h}, \bar{\rho}, \bar{v}$ - ratio of the density and the coefficient of kinematic viscosity of the gas and liquid, $\bar{v}=u_{*}^{g} / u_{*}, \bar{h}$ - ratio of the characteristic scale of gas layer to $l, \bar{\beta}_{3}=\varepsilon, \bar{\beta}_{6}=(1-1 / \bar{\rho}) \varepsilon /(\bar{U} C a), \bar{U}=\lambda_{U} / u_{*}^{2}$. The local mass flux of vapor at the interface $J_{e v}$ is determined by the Hertz-Knudsen equation [2]: $J_{e v} \quad=\left.\alpha_{J} T\right|_{z=h(x, t)}$., where $\alpha_{J}=\alpha \rho_{s} \lambda_{U} T_{*} / J_{*}\left(M /\left(2 \pi R_{g} T_{s}^{3}\right)\right)^{1 / 2}, \alpha$ is an accommodation coefficient, $\rho_{s}$ is the vapor density, $J_{*}-$ is the characteristic value of the vapor mass flux, $M$ is the molecular weight, $R_{g}$ is the universal gas constant, $T_{s}$ - saturated vapor temperature.

The no-slip conditions for velocity and the non-uniform heating are given on the solid plate :

$$
\left.u\right|_{z=0}=0,\left.\quad w\right|_{z=0}=0 .
$$




\section{The thin layer equation: scheme of the numerical solution}

The problem of finding of the unknown functions (longitudinal and transverse velocities, pressure and temperature) will be solved after obtaining the layer thickness $h$. Let us consider the problem statement for the zero-th order terms of the expansion in powers of $\varepsilon$ of the input functions. The following evolution equation determines the interface position (a consequence of the kinematic condition) [7]:

$$
\begin{gathered}
h_{t}+h_{x}\left\{\frac{h^{4}}{24} \gamma_{2} \cos \alpha A_{x}+\frac{h^{3}}{6}\left(\gamma_{2} \cos \alpha\left(\Theta_{0}\right)_{x}+\gamma_{1} \sin \alpha A\right)+\frac{h^{2}}{2}\left(\left(C_{0}\right)_{x}+\gamma_{1} \sin \alpha \Theta_{0}\right)+C_{1} h\right\}+ \\
+\left\{\frac{h^{5}}{120} \gamma_{2} \cos \alpha A_{x x}+\frac{h^{4}}{24}\left(\gamma_{2} \cos \alpha\left(\Theta_{0}\right)_{x x}+\gamma_{1} \sin \alpha A_{x}\right)+\right. \\
\left.+\frac{h^{3}}{6}\left(\left(C_{0}\right)_{x x}+\left(\Theta_{0}\right)_{x} \gamma_{1} \sin \alpha\right)+\frac{h^{2}}{2}\left(C_{1}\right)_{x}\right\}+\frac{E \alpha_{J}}{\varepsilon}\left\{A h+\Theta_{0}\right\}=0 .
\end{gathered}
$$

Here the function $\Theta_{0}$ defines the heating of the solid substrate, functions $A, C_{0}$ and $C_{l}$ are determined from the following relations:

$$
\begin{gathered}
A=\frac{\left(\bar{\beta}_{3} \alpha_{J}+\bar{\beta}_{6} h_{x x} \alpha_{J}\right) \Theta_{0}}{1-\bar{\beta}_{3} \alpha_{J}-\bar{\beta}_{6} h_{x x} \alpha_{J} h}, \\
C_{0}(x, t)=p^{g}-\alpha_{C a} h_{x x}\left(1-\alpha_{\sigma} \Theta^{0}\right)-\gamma_{2} \cos \alpha\left(A \frac{h^{2}}{2}+\Theta_{0} h\right)-\frac{\gamma_{1}}{B u} x \sin \alpha+\gamma_{2} h \cos \alpha, \\
C_{1}(x, t)=\alpha_{\tau} \tau(x, t)-\alpha_{M a} \tilde{\Theta}-\left(C_{0}\right)_{x} h-\gamma_{1} \sin \alpha h\left(A \frac{h^{2}}{2}+\Theta_{0} h\right)-\gamma_{2} \cos \alpha\left(A_{x} \frac{h^{3}}{6}+\left(\Theta_{0}\right)_{x} \frac{h}{2}\right) .
\end{gathered}
$$

Here $\tilde{\Theta}=A_{x} h+\left(\Theta_{0}\right)_{x}+h_{x} A, \Theta^{0}=A h+\Theta_{0}$.

The periodic problem of finding the function $h$ satisfying the equation (1) on the interval $[-L ; L]$ is considered. The following periodic conditions on the boundaries $x=-L, x=L$ are fulfilled:

$$
\left.h\right|_{x=-L}=\left.h\right|_{x=L},\left.\quad h_{x}\right|_{x=-L}=\left.h_{x}\right|_{x=L},\left.\quad h_{x x}\right|_{x=-L}=\left.h_{x x}\right|_{x=L},\left.\quad h_{x x x}\right|_{x=-L}=\left.h_{x x x}\right|_{x=L} .
$$

After substitution of expressions (2) and (3) the equation (1) can be rewritten as follows:

$$
h_{t}+A_{4} h_{x x x}+A_{3} h_{x x x}+A_{2} h_{x x}+A_{1} h+D=0 \text {. }
$$

The coefficients $A_{4}, A_{3}, A_{2}, A_{1}, D$ are the functions which depend on $A, h, \Theta_{0}$ and their derivatives. Equation (4) is solved numerically by using the following implicit finite-difference scheme [13]:

$$
\frac{h^{k+1}-h^{k}}{\tau}+A_{4}^{k} h_{x x x x}^{k+1}+A_{3}^{k} h_{x x x}^{k+1}+A_{2}^{k} h_{x x}^{k+1}+A_{1}^{k} h^{k+1}+D^{k}=0 .
$$

The scheme (5) is solved with use of the finite-difference analogues of the second-order approximation for all derivatives with respect to $x$. The uniform finite-difference mesh $x_{1}, x_{2}, \ldots, x_{N+1}$ $\left(x_{n}=-L+(n-1) \Delta x, \Delta x=2 L / N\right)$ is used for implementation of the scheme (5).

The scheme leads to a system of linear algebraic equations which can be solved by using the fivepoint Thomas algorithm and Thomas algorithm with parameter. The unknown value of the liquid layer thickness $h$ at $x=-L, x=L$ is chosen as the parameter.

Periodic movement of ethanol and HFE-7100 is studied numerically. The initial position of the thermocapillary boundary is given by the equation $h_{0}=1-\delta_{1} \cos k x$, where $\delta_{1}=0.01, k=\pi / 2$. Nonuniformly heating of the substrate is determined using the function $\Theta_{0}=1+\delta_{0} \cos k_{1} x \cdot \cos k_{2} t$, where $\delta_{0}=0.25, k_{1}=\pi / 2, k_{2}=2$.

Figure 2 presents the process of evaporation of the thin layer for the two liquids: HFE-7100 and ethanol in the case of low gravity $\left(g=9.81 \cdot 10^{-2} \mathrm{~m} / \mathrm{s}\right)$. The angle of inclination of the substrate $\alpha$ is equal to $\pi / 6$. Numerical investigation shows that the character of change of layer thickness is similar for different fluids but the quantitative results differ and the difference increases over time. 


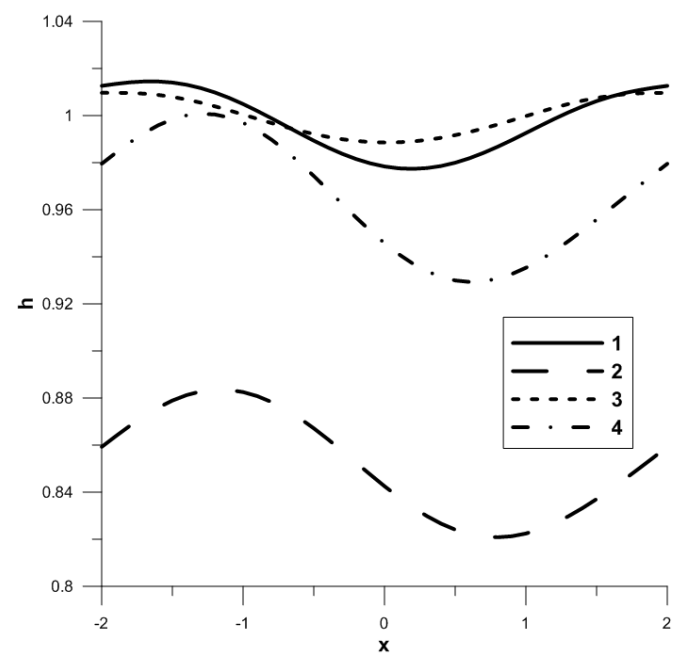

Figure 2. The behaviour of the liquid layer thickness, the case of low gravity $\left(g=9.81 \cdot 10^{-2} \mathrm{~m} / \mathrm{s}\right): 1-$ "HFE-7100nitrogen" system, $t=10^{-4}, 2$ - "HFE-7100-nitrogen" system, $t=10^{-2}, 3$ - "ethanol-nitrogen" system, $t=10^{-4}, 4-$ "ethanol-nitrogen" system, $t=10^{-2}$.

Acknowledgments. The research has been supported by the Russian Ministry of Education and Science (project indicator RFMEFI61314X0011).

\section{References}

1. A. Oron, S.H. Davis, S.G. Bankoff, Reviews of Modern Physics, 69 (3), 931-980 (1997)

2. S. Miladinova, S. Slavtchev, G. Lebon, J.-C. Legros, In: Proc. 6th Workshop on Transport Phenomena in Two-Phase Flow, Bourgas, 121-128 (2001)

3. Yu.O. Kabova, V.V. Kuznetsov, O.A. Kabov, International Journal of Heat and Mass Transfer, 55, 1271-1278 (2011)

4. Yu.O. Kabova, V.V. Kuznetsov, O.A. Kabov, T. Gambaryan-Roisman, P. Stephan, International Journal of Heat and Mass Transfer, 68, 527-541 (2014)

5. Yu.V. Lyulin, O.A. Kabov, Int. J. of Heat and Mass Transfer, 70, 599-609 (2014)

6. R.V. Craster, O.K. Matar, Review of Modern Physics, 81(3), 1131-1198 (2009)

7. O.N. Goncharova, E.V. Rezanova, Izvestia AltGU, 85 (1/1), 70-74 (2015)

8. O.N. Goncharova, E.V. Rezanova, Ya.A. Tarasov, Izvestia AltGU, 81 (1/1), 47-52 (2014)

9. O.N. Goncharova, E.V. Rezanova, Izvestia AltGU, 81 (1/2), 21-25 (2014)

10. C.S. Iorio, O.N. Goncharova, O.A. Kabov, Microgravity Sci. Technol, 21(1), 313-320 (2009)

11. C.S. Iorio, O.N. Goncharova, O.A. Kabov, Computational Thermal Sci., 3(4), 333-342 (2011)

12. O.N. Goncharova, Izvestia AltGU, 73 (1/2), 12-18 (2012)

13. E.V. Rezanova, Izvestia AltGU, 89 (1/1), 168-172 (2016) 